\title{
Thermal Annealing of Singlet Fission Microcrystals Reveals the Benefits of Charge Transfer Couplings and Slip-Stacked Packing
}

Zachary T. Armstrong, Miriam Bohlmann Kunz, Andrew C. Jones, Martin T. Zanni*

Department of Chemistry, University of Wisconsin, Madison, WI 53706

Correspondence to zanni@chem.wisc.edu

\section{Supporting Information:}

\section{Additional Transient Absorption Microscopy Images}

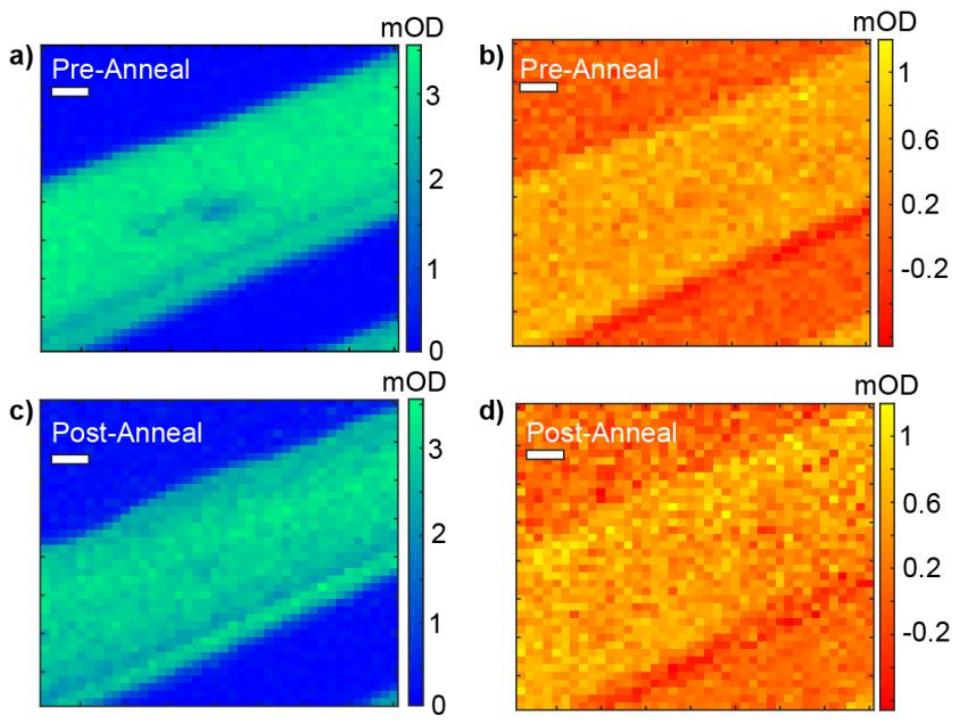

Figure S1.

Additional visualizations of the TAM data shown in Figure 3. Scale bars are $2 \mu \mathrm{m}$. a) Map of the intensity of the $S_{I, 0} \mathrm{GSB}$ before annealing. b) Map of the intensity of triplet ESA before annealing. c) Map of the intensity of the $S_{l, 0} \mathrm{GSB}$ after annealing at $90^{\circ} \mathrm{C}$. d) Map of the intensity of triplet ESA after annealing at $90^{\circ} \mathrm{C}$. 

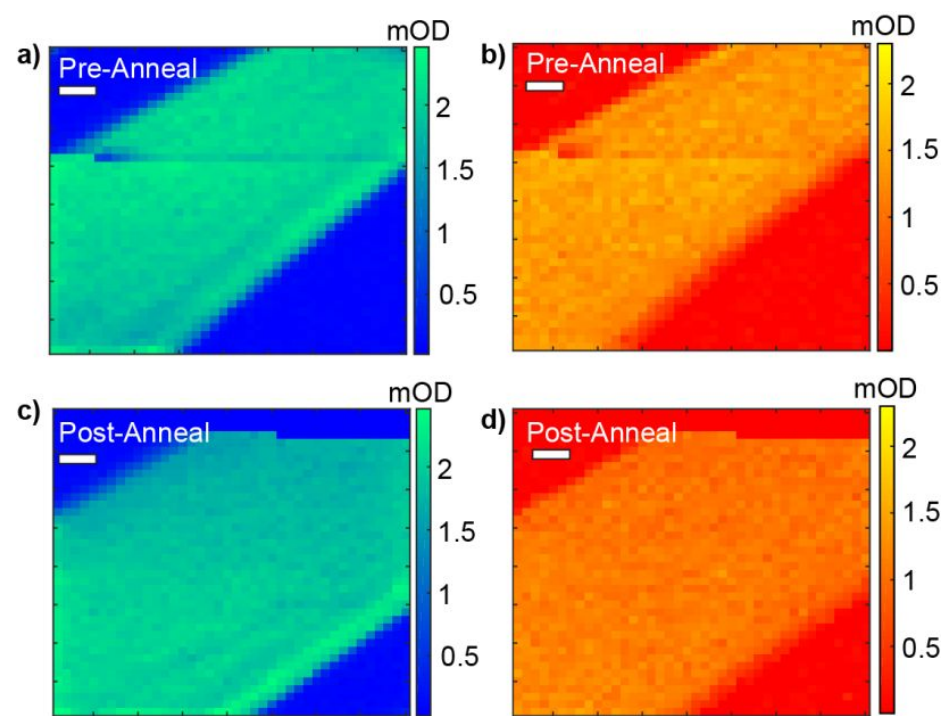

\section{Figure S2.}

Additional visualizations of the TAM data shown in Figure 5. Scale bars are $2 \mu \mathrm{m}$. a) Map of the intensity of the $S_{1,0}$ GSB before annealing. b) Map of the intensity of triplet ESA before annealing. c) Map of the intensity of the $S_{1,0}$ GSB after annealing at $90{ }^{\circ} \mathrm{C}$. d) Map of the intensity of triplet ESA after annealing at $90^{\circ} \mathrm{C}$.

\section{Comparison of $x$-ray diffraction data with known polymorphs of TIPS-pentacene}

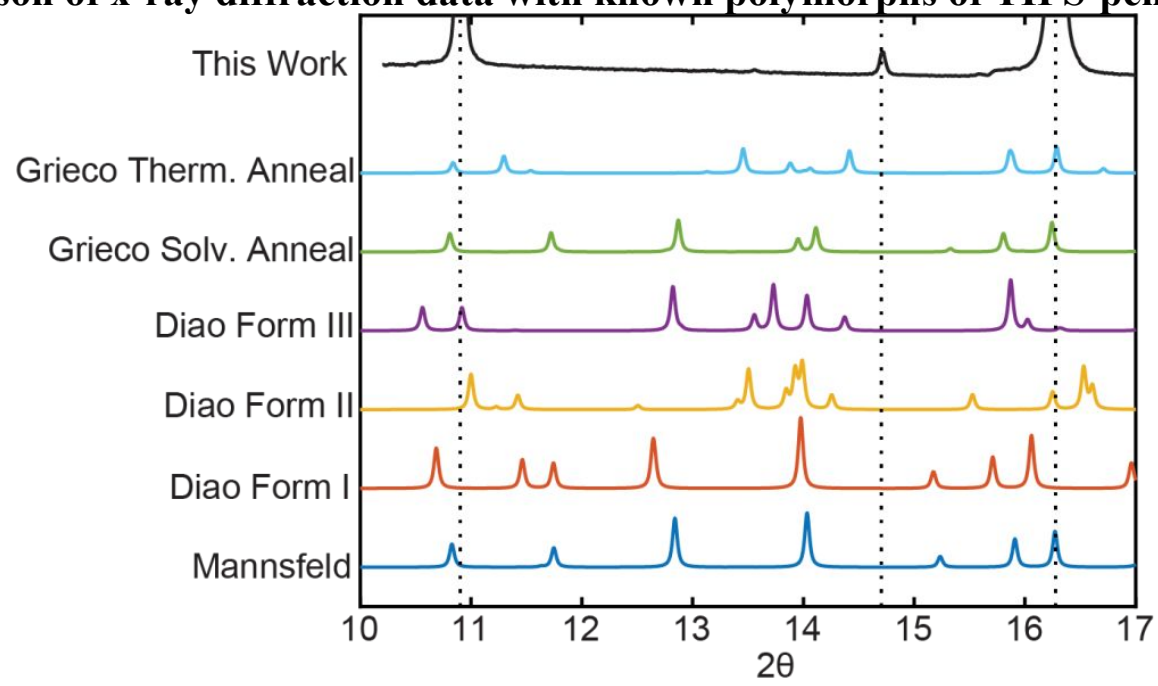

Figure S3.

Comparison of experimental x-ray diffraction patterns to patterns calculated in the Mercury software from previously published structures of TIPS-pentacene. ${ }^{1-3}$

\section{Derivation of Slip-Stacked Packing Concentration}

Experimental broadband transient absorption spectra were fit using the frequencies and transition dipoles of equilibrium and non-equilibrium models previously derived by Jones et al. Each spectrum in the TAM images were first sorted based on the intensity of the $S_{1,0}$ peak to determine which pixels were on the crystal and which pixels were over glass. Then, each pixel on the crystal was fitted with two Gaussians. The first 
Gaussian was fit to the $S_{1,0}$ peak, then subtracted from the experimental data and a second Gaussian was fit to the residual. Sequentially fitting these peaks allows for a more physically realistic estimate of the concentration of non-equilibrium packing, since we expect a majority of the crystal packing to be the equilibrium structure. The center frequencies of these two Gaussians were based off of the eigenstate energies derived from the dimer models but were allowed to vary slightly to account for disorder in the crystal. The concentration of non-equilibrium packing was calculated at each pixel by comparing the intensities of the two fitted Gaussians, correcting for differences in the transition dipole strengths of the equilibrium and non-equilibrium transitions. A representative fit is shown below.

\section{Figure S4.}

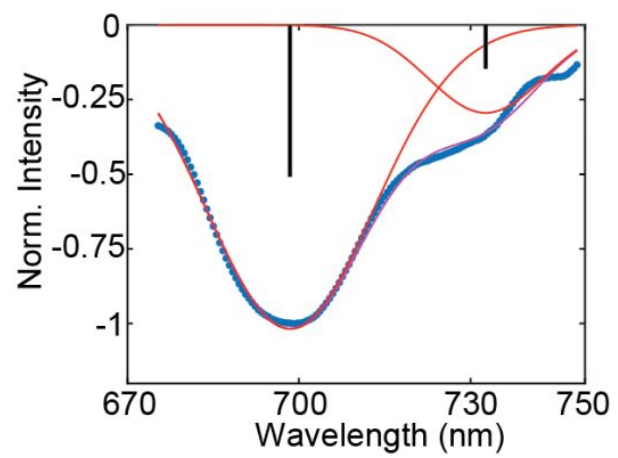

Representative fit to TAM data from Figure 3a.

\section{Atomic Force Microscopy}

We used atomic force microscopy to measure the topography of TIPS-pentacene microcrystals before and after thermal annealing. Overall, we found no detectable change in thickness or morphology of a range of crystals. However, we did observe an increase in the number of lateral cracks in the microcrystals as well as expansions of existing cracks. Representative AFM images are shown below in SI Figures 4-7.
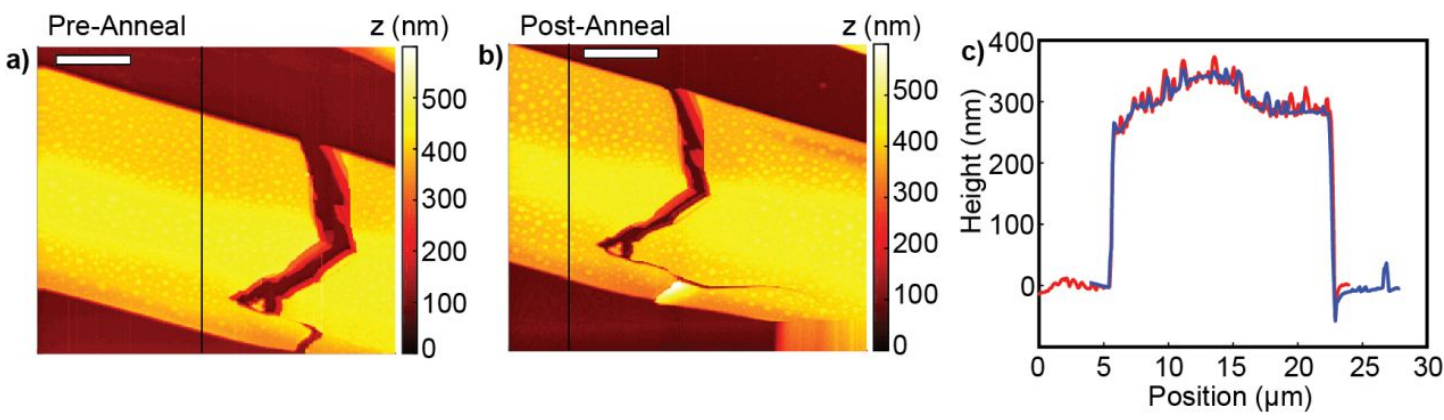

Figure S5.

AFM images before (a) and after (b) thermal annealing. Cuts along black line are shown in c. Scale bars are $5 \mu \mathrm{m}$. 

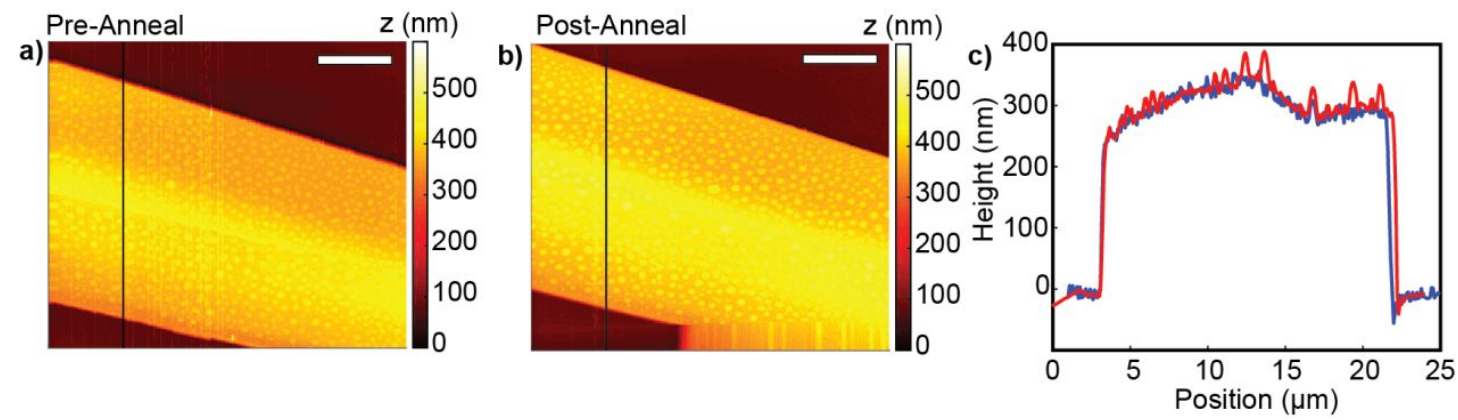

Figure S6.

AFM images before (a) and after (b) thermal annealing. Cuts along black line are shown in c. Scale bars are $5 \mu \mathrm{m}$.
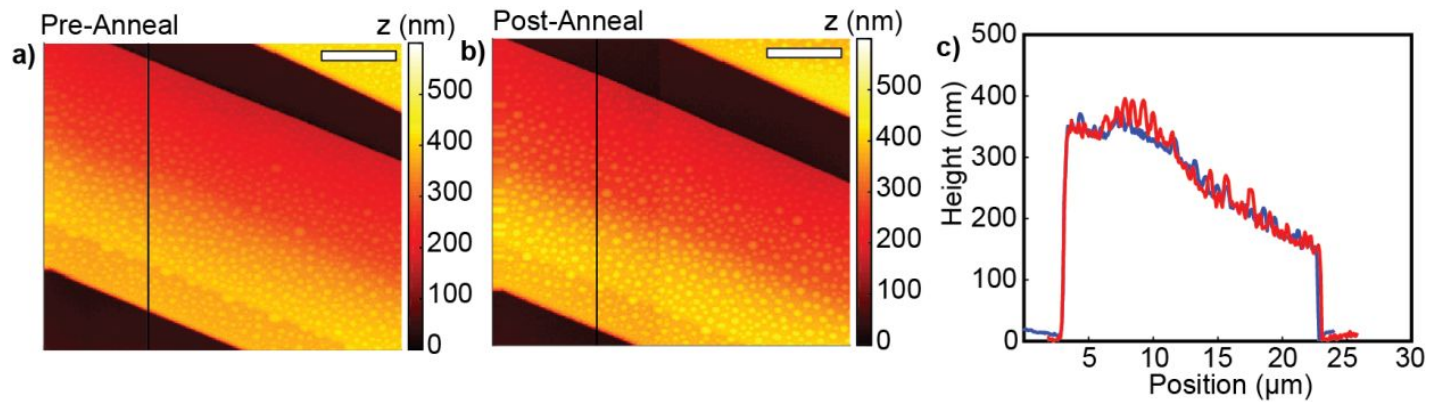

Figure S7.

AFM images before (a) and after (b) thermal annealing. Cuts along black line are shown in c. Scale bars are $5 \mu \mathrm{m}$.
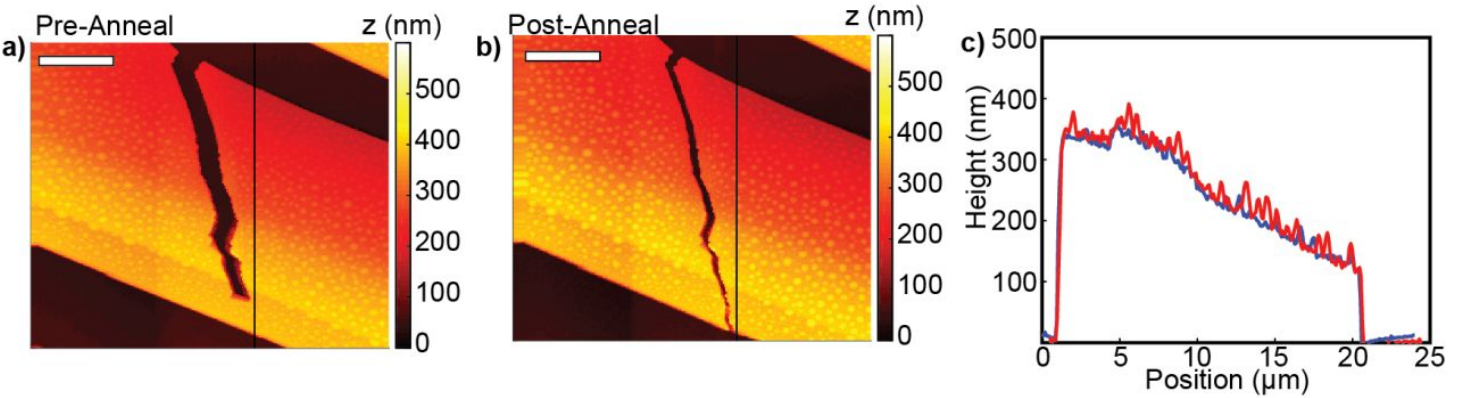

Figure S8.

AFM images before (a) and after (b) thermal annealing. Cuts along black line are shown in c. Scale bars are $5 \mu \mathrm{m}$. 


\section{References}

1 Mannsfeld, S. C. B.; Tang, M. L.; Bao, Z.; Thin film structure of triisopropylsilethynylfunctionalized pentacene and tetraceno[2,3-b]thiophene from grazing incidence x-ray diffraction Adv. Mater. 2011, 23, 127.

2 Grieco, C.; Doucette, G. S.; Munro, J. M.; Kennehan, E. R.; Lee, Y.; Rimshaw, A.; Payne, M. M.; Wonderling, N.; Anthony, J. E.; Dabo, I. et. al.; Triplet transfer mediates triplet pair separation during singlet fission in 6,13-Bis(triisopropylsilyethynyl)pentacene Adv. Funct. Mater. 2017, 27, 1703929.

3 Diao, Y.; Lenn, K. M.; Lee, W.; Blood-Forsythe, M. A.; Xu, J.; Mao, Y.; Kim, Y.; Reinspach, J. A.; Park, S.; Aspuru-Guzik, A. et. al.; Understanding polymorphism in organic semiconductor thin films through nanoconfinement J. Am. Chem. Soc. 2014, 136, 17046. 